\title{
Design of Sievenpiper HIS for use in planar broadband antennas by means of effective medium theory
}

\author{
S.K. Hampel, O. Schmitz, O. Klemp, and H. Eul \\ Leibniz Universität Hannover, Institut für Hochfrequenztechnik und Funksysteme, Appelstr. 9A, 30167 Hannover, Germany
}

\begin{abstract}
The claim for multistandard operating handsets of small physical size as well as the ever increasing demand for higher data rates require new broadband operating antennas. Because of the widespread use of especially planar broadband antennas a lot of factors influencing the characteristic antenna parameters have to be regarded. Furthermore, aspects regarding the electromagnetic compatibility inside the handheld as well as the protection of biological systems, e.g. the user of a mobilephone, have to be payed attention to. An electromagnetic structure which allows for protection by means of shielding as well as enhances the antennas efficiency by providing unique electromagnetic properties are the so called Sievenpiper High Impedance Surfaces (HIS) invented by Sievenpiper (1999). This paper will present the theory and the well known design equations for those structures. An investigation by means of simulation tools and measurement setups will be done to approve the accuracy of the theoretical results. Here measurement results of the impedance and radiation properties of a planar log.-per. fourarm antenna equiped in conjunction with a fabricated prototype Sievenpiper HIS will be presented.
\end{abstract}

\section{Introduction}

The trend of digital convergence with its multiradio applications keeps on raising the bar for research and application engineers. On the one hand more spectral effiency and interoperability is demanded by the customers by simultaneously needs for increasing battery life and decreasing physical size of the handheld. On the other hand the complexity of the transceiver circuitry and with it the production costs raise due to operating in different communication standards with multiple antennas. Therefor it is necessary to use broad-

Correspondence to: S. K. Hampel

(hampel@hft.uni-hannover.de) band antennas with an easy impedance matching network and constant antenna parameters regarding frequency, e.g. input impedance or radiation pattern, to keep circuitry complexity down. An antenna which provides such properties is for example the so called planar log.-per. trapezoidal antenna (TA) as proposed in Klemp et al. (2005) which is a representative for the group of frequency independent antennas. These planar broadband antennas exhibit a bidirectional radiation characteristic which can cause perturbation due to parasitic effects such as surface wave propagation or electromagnetic wave interaction with different sections of a handset. Because of that volumetric effects there is a need for so called spatial filters to ease the perturbation occuring in the communication terminal while conserving the radiation efficiency of the antenna as well as shielding the environment against unwanted radiation.

The Sievenpiper HIS offers attractive boundary conditions to overcome the mentioned obligations. This type of spatial filter is a metallo-dielectric material with $2 \mathrm{D}$ periodicity where all physical sizes of a so called unit cell must be much smaller than the corresponding wavelength in the operational bandwidth. In general the structure consists of periodic cylindrical vias placed in a grounded dielectric slab with a metal frequency selective surface layer (FSS) on top.

Because of combining the reflection properties of artificial magnetic conductors (AMC) and the ability of suppressing surface wave propagation like electromagnetic band-gap structures (EBG) (see Sievenpiper, 2003) this structures seem to be adequate for use in mobile communication handhelds. The resulting reflection phase of $\phi_{\Gamma}=0^{\circ}$ with an absolute reflection coeficient of $|\Gamma|=1$ at the surface allows using the HIS as near field reflector while incident and reflected wave will not cancel out each other. Due to the in-phase image current the close proximity of the Sievenpiper HIS reflector to radiating elements results in low profile antennas with an enhanced efficiency compared to fully metallic reflectors or absorber materials. Furthermore, the suppression of surface

Published by Copernicus Publications on behalf of the URSI Landesausschuss in der Bundesrepublik Deutschland e.V. 


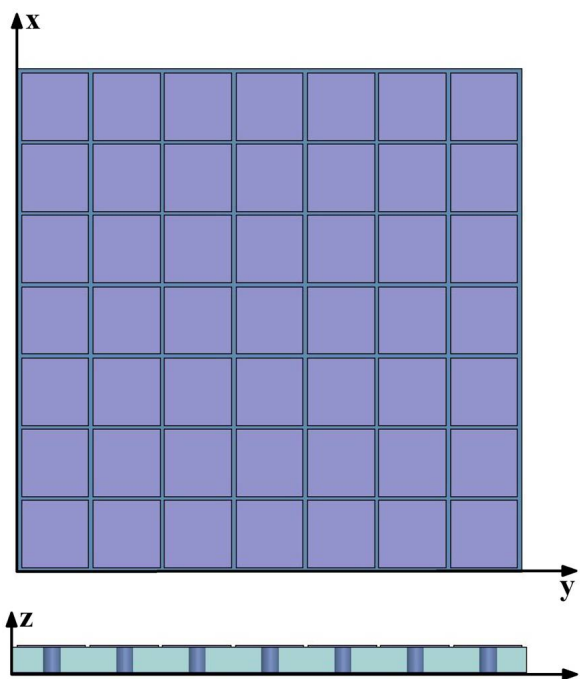

Fig. 1. Sievenpiper HIS placed in the $x y$-plane displayed in topand side-view.

waves eliminates the transport of interfering power to circuitry elements in the transceiver which can cause malfunctions. Moreover a smooth and uneffected radiation pattern can be expected because of avoiding parasitic radition from finite groundplane edges.

The organization of the paper is as follows. In Sect. 2 the underlying theory of the Sievenpiper HIS and the equations for designing the geometry parameters of the structure for a given frequency of operation are presented. Section 3 deals with simulation models and simulation results for verifying the derived equations. Here the simulated reflection phase properties for orthogonal incidence and the influence of the HIS on matching and radiation properties of a reference dipole are presented. In the last section measurement results of a planar broadband log.-per. four arm trapezoidal antenna in conjunction with a fabricated prototype Sievenpiper HIS are highlighted. The properties under consideration are the input impedance as well as the radiation pattern.

\section{Theoretical description of reflection properties}

The underlying theory of Sievenpiper HIS is in general based on the effective medium theory (see Merill, 1999) and moreover on the transmission line theory. These theories can be used when describing the (homogenized) electromagnetic behaviour of small inclusions in a host material or the quasistatic properties of devices much smaller than the wavelength respectively. On the one hand the structures can be seen as composite materials consisting of a periodic FSS on a conductor backed substrate, acting as a boundary with such a high surface impedance, leading to a vanishing magnetic field in theory, therefore called artifificial magnetic conductor (AMC).

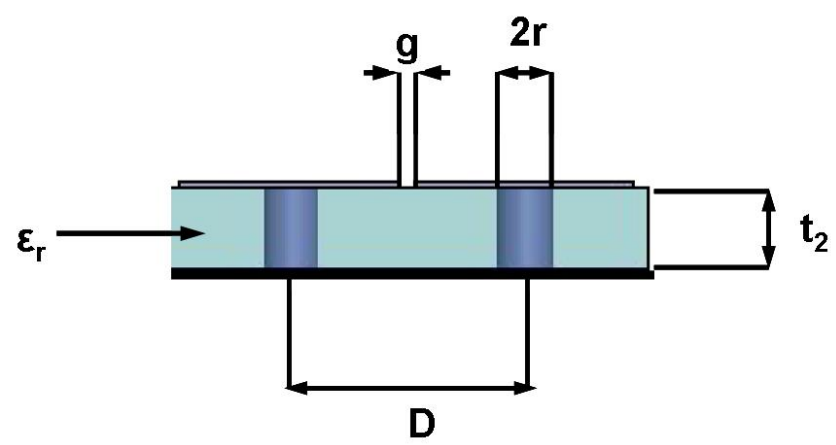

Fig. 2. Side-view of a zoomed region of the Sievenpiper HIS from Fig. 1 containing two unit cells with each a size of $D$.

On the other hand the cylindrical vias embedded periodically in the substrate build the so called Brown's rodded medium or the "Fakir's bed of Nails" which solution can be found in Rotman (1962) and King et al. (1983). They are acting like a plasma below its plasma frequency, suppressing the propagation of surface waves comparable to the double negative materials (DNG) or metamaterials (MM), first mentioned by Veselago (1968). Because of exhibiting frequencies where no wave propagation is possible a so called band-gap occurs. Due to that textures which offer this kind of filter mechanism they are called electromagnetic band-gap structures (EBG).

Due to the very complex mechanisms yielding to the surface wave suppression and the complications in finding adequate equations describing the interaction of electromagnetic waves and the periodic structure, here only the reflection properties are highlighted. Furthermore, the propagation of surface waves in single antenna stand-alone applications is less critical than in antenna array arrangements and will be neglegted in a first approximation. Further information about and insight into the surface wave properties of these structures can be found in Clavijo (2003) and Sievenpiper (1999).

For determining the reflection properties of the exemplary structure in Fig. 1 the transmission line theory can be used, no matter what kind of patch geometry will be offered by the FSS (triangle, square, hexagon,...). The only restriction is the physical size of the geometry with regard to the corresponding wavelength at the operational frequencies. All describing parameters of a so called unit cell shown in Fig. 2 as substrate-heigth $t_{2}$, via-radius $r$, periodicity $D$ or gapwidth $g$ have to be much smaller than the wavelentgh, i.e. $t_{2}, r, D, g<<\lambda$. For an orthogonal incident wave travelling in negative $z$-direction adjacent edges of the FSS build a shunt capacitance. The resulting admittance of two adjacent metal stripes seen by the wave, can be obtained by means of calculus of variations and is well known in literature (see Marcuvitz, 1996). Calculating the edge capacitance from this 


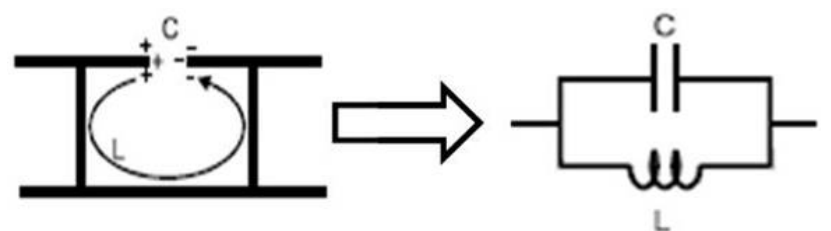

Fig. 3. Equivalent LC circuit for a unitcell.

admittance leads to

$C_{G_{T M}}=\epsilon_{e f f} \epsilon_{0} \frac{2 D}{\pi} \ln \left(\frac{2 D}{\pi g}\right)$

for TM polarization and

$C_{G_{T E}}=\epsilon_{e f f} \epsilon_{0} \frac{2 D}{\pi} \ln \left(\frac{2 D}{\pi g}\right) \cos ^{2} \theta_{i}$

for TE polarization (see Simovski, 2005). Here $\epsilon_{\text {eff }}=\sqrt{\left(\epsilon_{1}+\epsilon_{2}\right) / 2}$ indicates the effective permittivity of the two half spaces under consideration $(z<0, z>0)$ and $\theta_{i}$ is the angle of incidence.

The conductor backed host-medium itself offers an inductive surface impedance, which is typical for substrate layers as can be found in Collin (1991) for example. The via array stabilizes the surface impedance of the substrate by mimicing a TEM waveguide for TM polarized waves. The expression for the surface impedance for both TE and TM polarization is therefore equivalent and as a first approximation independant of the incidence angle (see Clavijo, 2003). This yields to:

$Z_{D_{T E}}=Z_{D_{T M}}=j \omega \mu_{0} t_{2}$

Both surface impedances are in parallel for an incident wave and can be combined by means of the well known equation for parallel resistors in lumped networks expressed in Eq. (4).

$Z_{S}(\omega)=\frac{Z_{G}(\omega) Z_{D}(\omega)}{Z_{G}(\omega)+Z_{D}(\omega)}$

For orthogonal incidence as well as for small angles of incidence the capacitive impedance for the FSS layer is given by $Z_{G_{T M}}=Z_{G_{T E}}=Z_{G}=1 / j \omega C_{G}$. While neglecting losses the two impedances build an ideal parallel resonant circuit as shown in Fig. 3. At resonant frequency the denominator vanishes and the impedance goes for infinity. The equation for the reflection coefficient known from the transmission-line theory (see Zinke and Brunswig, 2000) by use of the derived expressions for the resulting surface impedance yields

$r=\frac{j \omega t_{2} \mu_{r} \mu_{0}-Z_{0}\left(1-\left(\frac{\omega}{\omega_{0}}\right)^{2}\right)}{j \omega t_{2} \mu_{r} \mu_{0}+Z_{0}\left(1-\left(\frac{\omega}{\omega_{0}}\right)^{2}\right)}$

with $\omega_{0}=1 / \sqrt{L C}$ representingthe resonant frequency of the circuit.

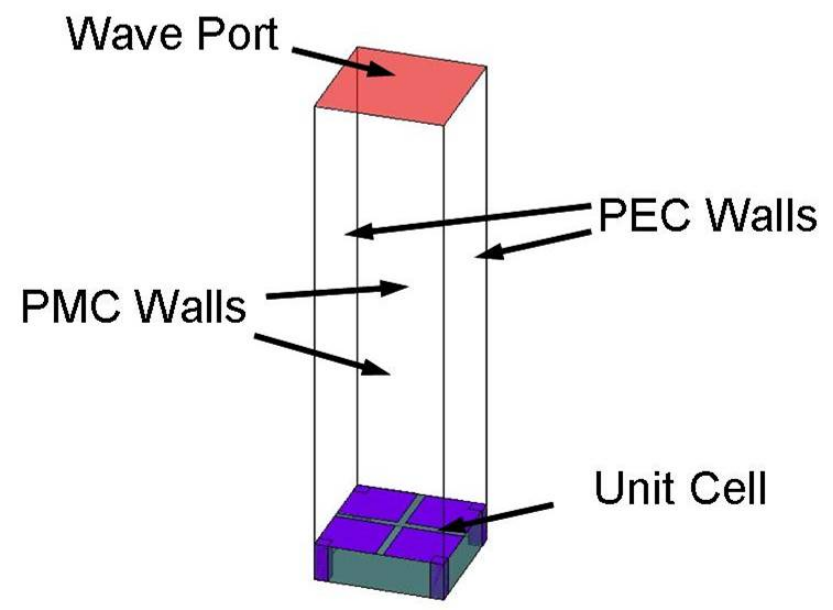

Fig. 4. HFSS simulation model for determining the reflection properties of a HIS: TEM waveguide with periodic boundary conditions.

Due to the metallic surface of the structure the absolute value of the reflection coefficient remains 1 , whereas the occuring reflection phase decides on whether more constructive or more destructive interference takes place. Because of the frequency dependence of the reflection coefficient an operational bandwidth can be given which is located in between the frequencies where the reflection phase $\phi_{\Gamma}$ equals $\pm \pi / 2$. Under this constraints and with the definition of a relative bandwidth $B_{r e l}=f_{u} / f_{l}$, the ratio of the upper frequency $\left(\Phi_{\Gamma}=-\pi / 2\right)$ to the lower frequency $\left(\Phi_{\Gamma}=+\pi / 2\right)$ of the operational bandwidth, Eq. (5) can be written as follows:

$\frac{B_{r e l}-1}{\sqrt{B_{r e l}}}=2 \pi \mu_{r} \frac{t_{2}}{\lambda_{\text {res }}}$.

Here $\lambda_{\text {res }}$ stands for the wavelength at resonant frequency. As can be seen, this transcendent expression and the bandwidth respectively depend mainly on the substrate height.

\section{Simulation results}

This section deals with simulation results for evaluating the derived design equations by means of the simulation tool $H F S S$ of Ansoft Corporation. First of all the reflection properties of a Sievenpiper HIS will be investigated. The used simulation model is shown in Fig. 4 and consists of an ideal TEM waveguide. This is realized by the use of so called periodic boundary conditions where adjacent walls offer perfect electric and perfect magnetic boundary conditions alternating circular around the longitudinal axis. The TEM wave is excited by a wave port traveling down the longitudinal axis of the waveguide. At the end a unit cell of the HIS under test is placed. The parameters of the designed HIS are $D=8.5 \mathrm{~mm}, g=0.5 \mathrm{~mm}, t_{2}=3 \mathrm{~mm}$ and $r=1 \mathrm{~mm}$. The host substrate is a FR4 with a relative permittivity of $\epsilon_{r}=4.4$ resulting in a theoretical resonant frequency of $f_{\text {res }}=4.65 \mathrm{GHz}$ 


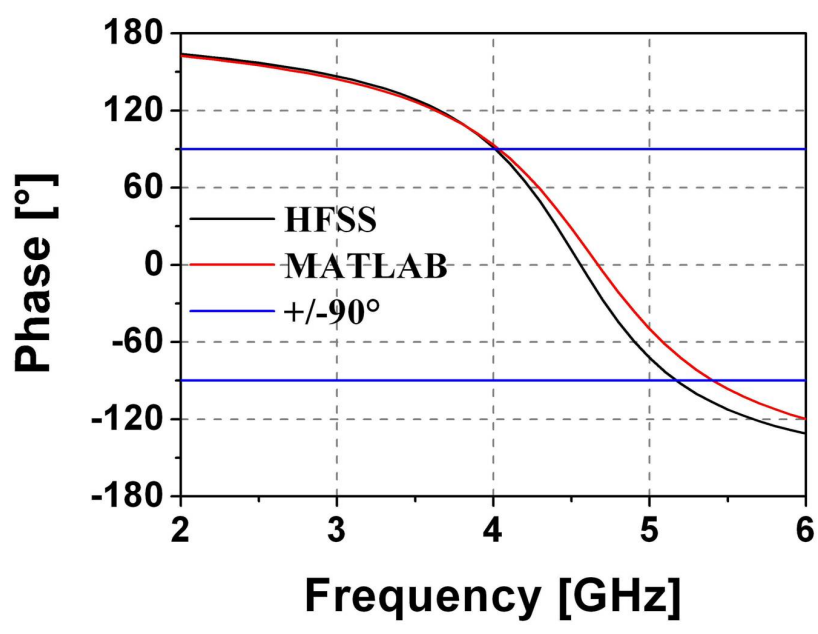

Fig. 5. Reflection phase of the considered HIS over frequency.

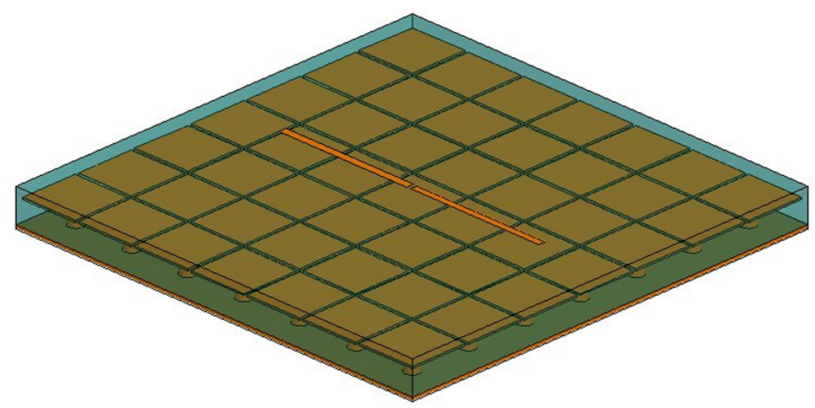

Fig. 6. Flat dipole at FR4 substrate placed $1.5 \mathrm{~mm}$ above a Sivenpiper HIS.

with bandedges at $f_{l}=4 \mathrm{GHz}$ and $f_{u}=5.2 \mathrm{GHz}$. The reflection phase of this structure can be easily calculated when using this simulation model by deembeding of the reflection coefficient of the wave port onto the surface of the HIS. The curves over frequency derived by the simulation and the presented equations from Sect. 2 implemented in MATLAB are shown in Fig. 5. A good agreement of the two curves can be observed. Only the resonant frequency and the frequency where $\Phi_{\Gamma}=-\pi / 2$ reveal small differences of $100 \mathrm{MHz}(2.2 \%)$ and $200 \mathrm{MHz}(3.9 \%)$ respectively. But under consideration of the simplifications done by deriving the equations these results can be seen as a proof for the accuracy of the design formulas for determining the reflection properties of a Sievenpiper HIS. The only drawback of this simulation model is the invariable orthogonal incidence of the propagating wave. Moreover due to the wide spectrum of different modes existing in the reactive near field of a radiating antenna and their corresponding incidence angles, small deviations from the desired functionality and operational frequency must be taken into account.

The next step while analyzing the fuctionality of the this

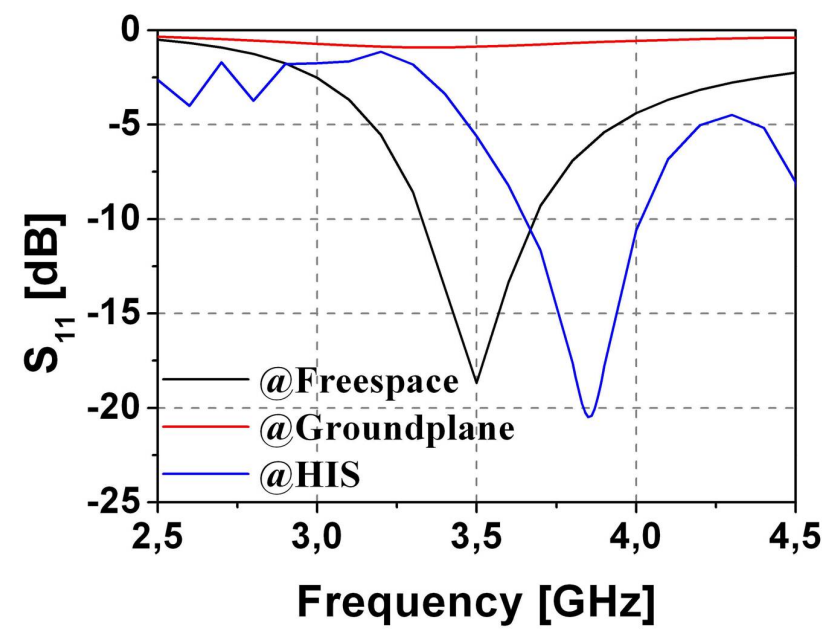

Fig. 7. Return loss for a flat dipole placed above a perfect conducting plate, above a HIS and in freespace.

geometry is placing an antenna above the structure and investigating the influence of the HIS on its characteristic properties and vice versa. For the purpose of being able to evaluate the effect of the Sievenpiper HIS on the antenna correctly it is important to use a simple and well known antenna geometry. Therefore the use of a dipole as reference antenna geometry is convenient and often used in literature while evaluating miscellaneous applications. Figure 6 shows the configuration of the dipole in conjunction with the filter structure.

Considered parameters for evaluating the efficiency of this arrangement and especially the HIS are the return loss of the antenna as well as its gain. The dipole length is designed for resonating at $3.5 \mathrm{GHz}$ and it is placed on a FR4 substrate with a thickness of $1.5 \mathrm{~mm}\left(\approx \lambda_{e f f} / 30 @ 3.5 \mathrm{GHz}\right)$.

Applying the same parameter values to the HIS structure as in the case of investigating the reflection properties the resonant frequency of the surface impedance is shifted down to $3.65 \mathrm{GHz}$ based on the derived equations and $3.55 \mathrm{GHz}$ resulting from the simulation. This shift is founded by placing the antenna substrate on top of the HIS which affects the capacity due to replacing the effective medium consisting of air and FR4 by a homogeneous medium consisting exclusively of FR4. This increases the shunt capacitance of adjacent edges and hence reduces the resonant frequency. Figure 7 displays the curves for the return loss. To be able to evaluate the composite structure of antenna and HIS the curves for the dipole placed in freespace as well as for the case of a conductor backed antenna substrate with $1.5 \mathrm{~mm}$ thickness are also stated. While regarding the run of the curve for the dipole in conjunction with the metallic groundplane it is obvious that this configuration does not accept or radiate any power respectively. The dipole at freespace reveals the expected characteristic with a minimum of $S_{11}=-18 \mathrm{~dB}$ at $3.5 \mathrm{GHz}$, the designed resonant frequency. The dipole placed above 
the Sievenpiper HIS offers almost the same characteristic as the arrangement in freespace does. The main difference is a shift of the whole curve up to higher frequencies with a better return loss of $S_{11}=-21 \mathrm{~dB}$ at $3.8 \mathrm{GHz}$. The frequency shift can be explained as follows. The dipole is excited by a so called lump port which mimics an ideal source arranged between the two arms of the dipole. The arms form a parasitic capacitance with the metallic surface of the HIS which is in parallel to the antenna arms. So the dipole seems to be electrically shorter and resonates at a higher frequency. Investigations with two coaxial cables as feeder for the dipole have shown the opposite effect of building a series resonance, due to the fact that the outer conductor was connected to the groundplane of the HIS. Moreover a variation of the antenna substrat-height shows a decreasing frequency of the point with minimal return loss, while increasing the substrate thickness. This can be regarded as reducing the parasitic parallel capacitance which is assumed to be proportional to the inverse of the substrate height (comparable to a parallel-plate capacitor).

Furthermore the phase shift of a wave travelling through the antenna substrate and back has to be regarded. So the optimal constructive interference of the radiated and reflected wave has to be expected for frequencies where $\phi_{\Gamma}<0^{\circ}$, which is above the resonance of the Sievenpiper HIS. The discrepancies of the two curves above $4.25 \mathrm{GHz}$ is related to an occuring edge resonance of the HIS groundplane at about $4.65 \mathrm{GHz}$. The corresponding characteristic of the absolut gain in the main direction, i.e. in $z$-direction, is presented in Fig. 8.

Due to the results for the return loss the curve of the dipole placed $1.5 \mathrm{~mm}$ over a metal plate will not be analyzed here because as mentioned before this arrangement does not accept any power and hence can not radiate effectively.

The dipole in freespace reveals an almost constant gain over frequency. At the point of minimum return loss the absolut gain is $G_{i}=2.5 \mathrm{~dB}$ which is in good agreement with the theoretical result for an ideally thin wire dipole which offers $G_{i}=2.15 \mathrm{~dB}$.

As can be seen in the frequency range of good matching, i.e. $S_{11}<-10 \mathrm{~dB}$, from $3.6 \mathrm{GHz}$ to $4 \mathrm{GHz}$ the absolute gain of the dipole in conjunction with Sievenpiper HIS is much higher than for the dipole in freespace. This observed effect can be seen as a proof for the predicted reflection properties of the HIS since the in-phase reflection of an incident wave leads to constructive interference. This superimposition of a directly radiated wave and the reflected wave ideally doubles the electric field strength in the primary half space of radiation and hence leads to an increase in absolute gain of $3 \mathrm{~dB}$. This effect can be observed in Fig. 8 at the frequency point with best matching at $3.8 \mathrm{GHz}$. Here the value for the absolute gain is $G_{i_{3.8 \mathrm{GHz}}}=5.8 \mathrm{~dB}$. The boost of the gain by a value higher than $3 \mathrm{~dB}$, exactly $3.3 \mathrm{~dB}$, is founded in numerical uncertainty.

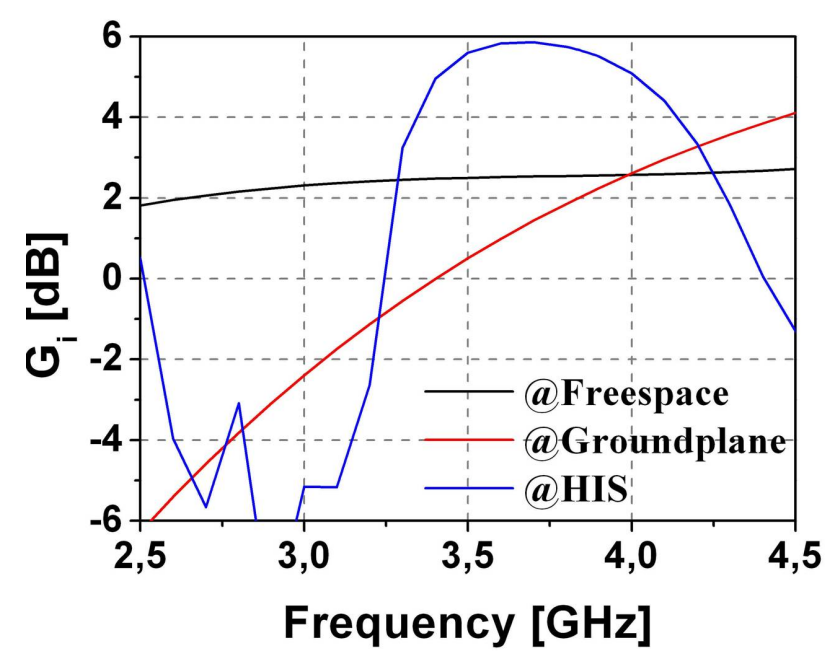

Fig. 8. Absolut antenna gain in the main direction for a flat dipole placed above a perfect conducting plate, a HIS and in freespace.

Finally the radiation patterns of the two arrangements in freespace and over the Sievenpiper HIS are evaluated. Figure 9 shows both the horizontal and the vertical diagramm of the dipole for the two dispositions. Here again the improvement of the gain is obvious. Furthermore it can be seen that the front to back ratio is increased due to the shielding effect of the HIS structure with respect to the lower half space. By the way the smooth pattern is an indication for no or at least negligible parasitic radiation from the HIS structure or its groundplane which normally generates sidelobes in the pattern.

After having highlighted the correct function of the HIS and the accuracy of the design equations, now an evaluation by means of measurement results will be done.

\section{Measurement results}

The former section which dealed with simulation results revealed the accuracy of the derived equations of Sect. 2 for designing Sievenpiper HIS with respect to its reflection properties. The next step treated in this section is to fabricate a prototype and measure its influence on important antenna parameters of a planar broadband antenna, while using the HIS as near field reflector, as done before in the simulation.

The used braodband antenna is the so called planar log.per. four-arm trapezoidal antenna (TA) with $\widehat{w}=45^{\circ}$ and $M=5$ periods as presented in Klemp et al. (2005).

The mentioned trapezoidal antenna is depicted in Fig. 10.

Due to its four arms this antenna geometry offers the possibility of dual linear or circular polarized radiation. For dual linear polarization two opposite arms are excited in antiphase. Hence differential excitation of adjacent arm pairs with no phase difference between the upper and right (or left) 

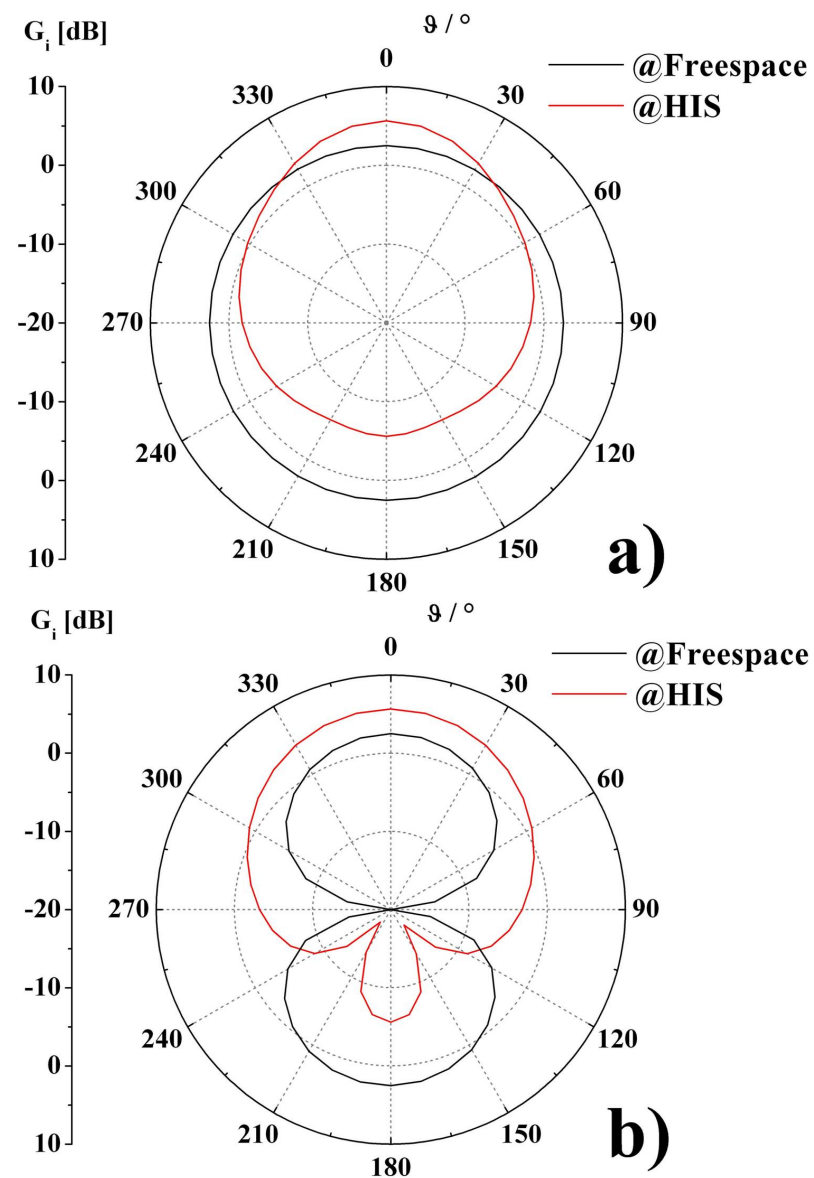

Fig. 9. Horizontal and vertical dipole characteristic for arrangement in freespace and in conjunction with HIS for cuts at a) $\phi=0^{\circ}$ and b) $\phi=90^{\circ}$.

arm and the lower and left (or right) arm results in a radiating configuration which is similar to a cross dipole. Attention has to be paid to the fact that horizontal excitation yields vertical polarized radiation and vice versa due to the shape of the arms.

Another main feature of this antenna is its quasi-frequency independent input inpedance as well as the almost constant radiation properties like gain and effective antenna area respectively due to its selfcomplemetarity and selfsimilarity. The theoretical values are known from literature and former investigations and publications (see Klemp et al., 2005) and must be constant over frequency in the range of approximately $2 \mathrm{GHz}$ up to $6 \mathrm{GHz}$ for the regarded standard TA.

The antenna was fabricated on $1.5 \mathrm{~mm}$ FR4 which results in an approximately purely real input impedance of $\operatorname{Re}\left\{Z_{i n}\right\}=77 \Omega$ and a vanishing imaginary part.

The prototype of the Sievenpiper HIS used as near field reflector in conjunction with the introduced planar broadband antenna offers the same parameters as stated in the simulation model which yields a theoretical resonant frequency of

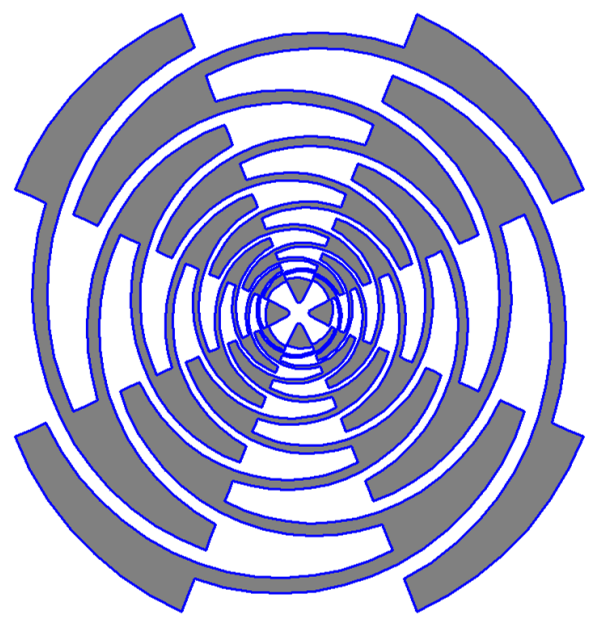

Fig. 10. Standard planar broadband four arm log.-per. trapezoidal antenna.

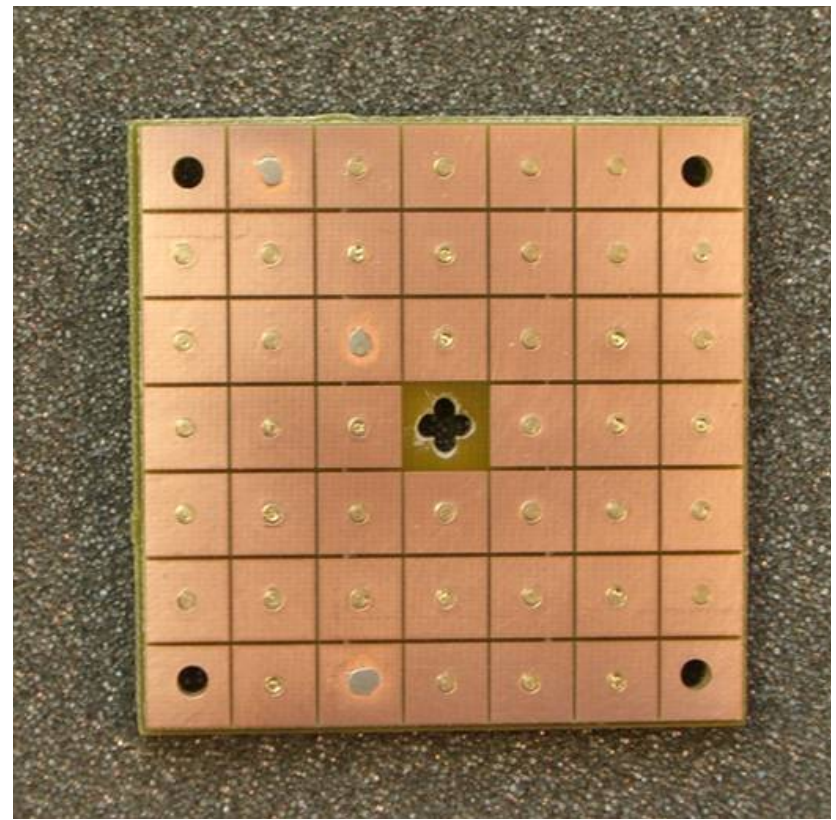

Fig. 11. Fabricated prototype of Sievenpiper HIS with theoretical resonance frequency at $f_{\text {res }}=3.65 \mathrm{GHz}$ and a relative bandwidth of $B_{\text {rel }}=1.26$ ranging from $f_{l}=3.25 \mathrm{GHz}$ to $f_{u}=4.1 \mathrm{GHz}$.

$f_{\text {res }}=3.65 \mathrm{GHz}$ in homogeneous FR4 substrate with an permittivity of approximately $\epsilon_{r}=4.4$. A picture of the prototype is shown in Fig. 11 with drill holes in the center for the four semi ridgid feed lines of the antenna.

The characteristic of the real part as well as the imaginary part of the input impedance of opposite arms excited in a differential way is plotted in Fig. 12. As in the simulation of the reference dipole, here the curves for the TA on a grounded dielectric FR4 slab, on a microwave absorber as well as on the fabricated HIS are shown over frequency. 

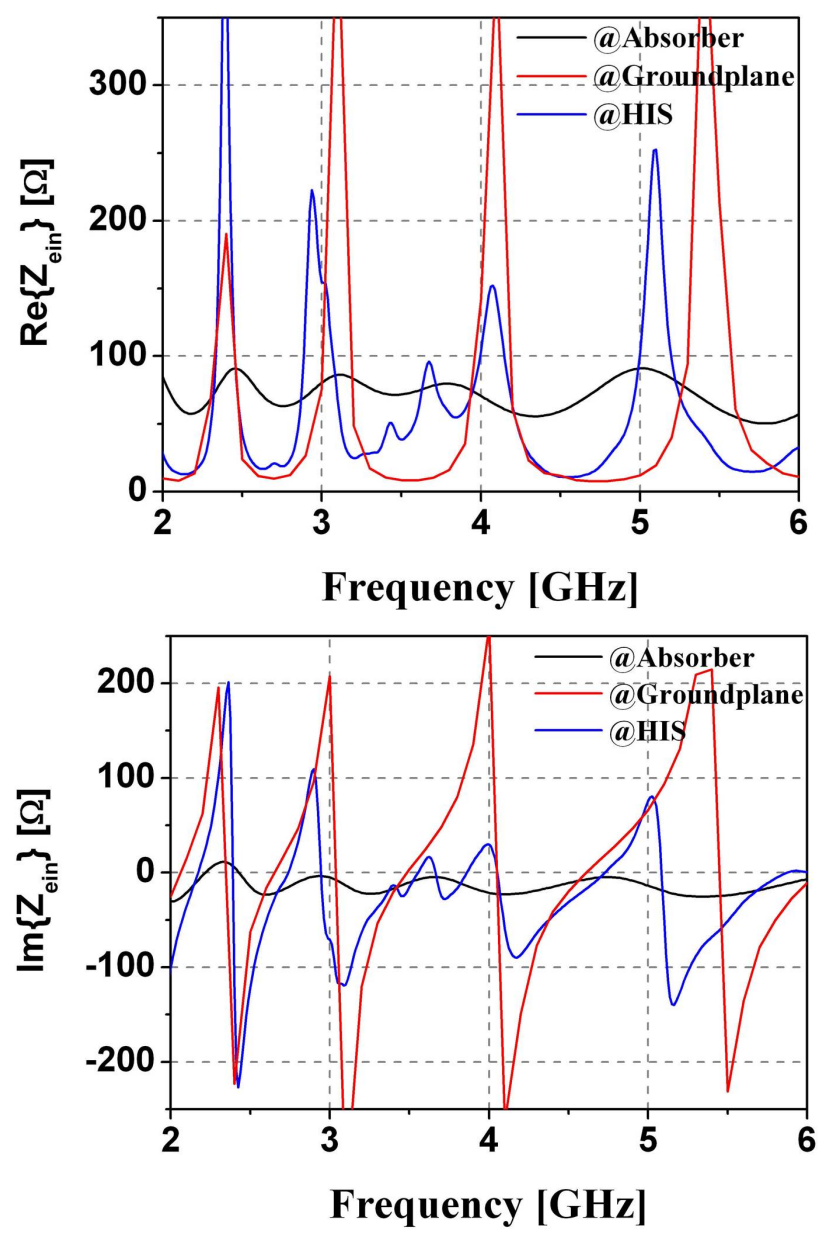

Fig. 12. Real and imaginary part of the input impedance over frequency of one arm pair for the TA in conjunction with a metal plate, a microwave absorber as well as the fabricated HIS.

The curve for the TA on the conductor backed substrate reveals strong resonance behaviour with no remarkable matching point in both real and imaginary part. When considering the characteristics of the impedance, while using the filter structure, a strong effect of the HIS becomes obvious between $3 \mathrm{GHz}$ and $4 \mathrm{GHz}$. This effect is expressed in variations from the resonant behaviour occuring outside this frequency band. Further investigations between $3.5 \mathrm{GHz}$ and $4 \mathrm{GHz}$ show similarities between the reference curve when using a microwave absorber and the one with the near field reflector. The imaginary part vanishes, whereas the real part alternates with an amplitude of only $20 \Omega$ around a theoretical (virtual) mean value of $77 \Omega$ offered by the curve while using the microwave absorber. An optimal match is observed at $3.6 \mathrm{GHz}$ which coincides with the theoretical predicted behaviour of the HIS structure.

Now the influence of the reflector on the radiation characteristic at a specific frequency point will be highlighted. As mentioned before a quasi optimal match occurs at $3.6 \mathrm{GHz}$.
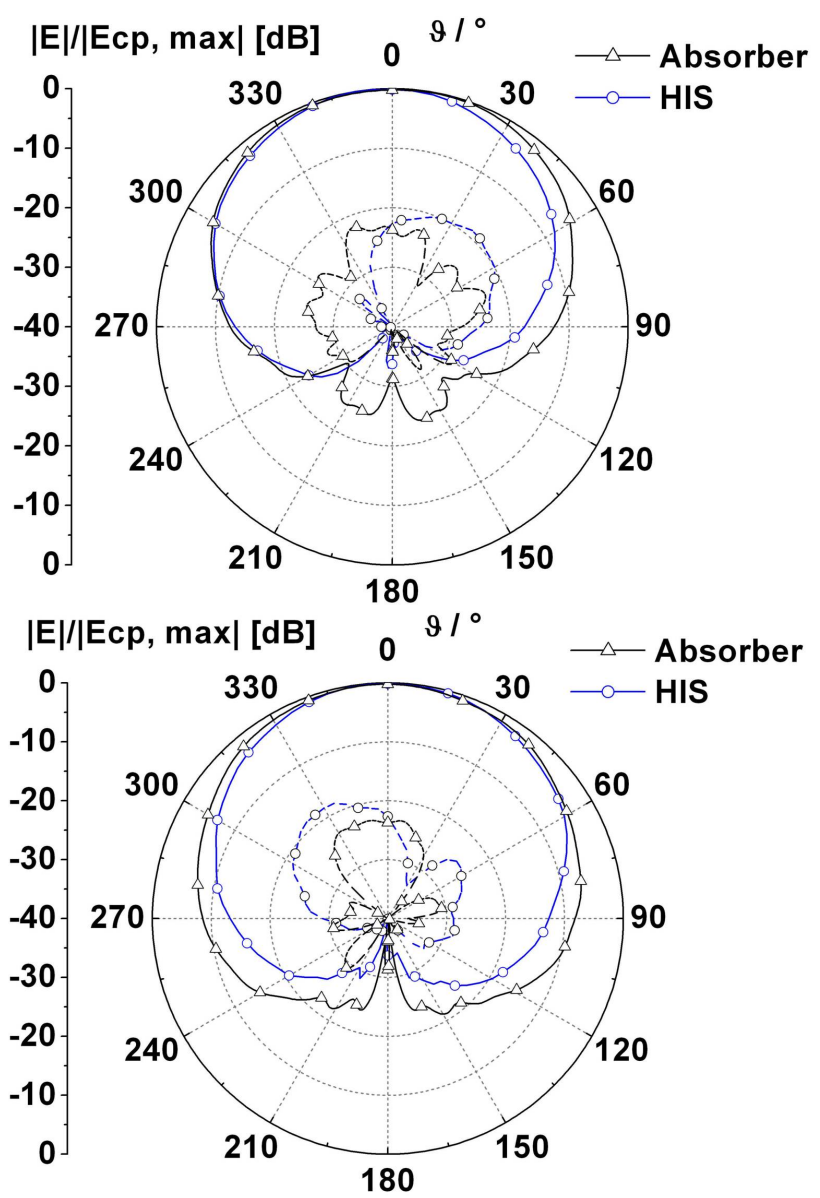

Fig. 13. Horizontal and vertical diagramm of the relative radiation pattern for the TA in conjunction with a microwave absorber and a Sievenpiper HIS at $3.6 \mathrm{GHz}$.

Therefore it is straightforward to evaluate the radiation pattern at this frequency. The resulting pattern for the TA positioned at a microwave absorber as well as over the HIS prototype are presented in Fig. 13.

The measured characteristics reveal the benefit when using the HIS structure. It is obvious that the Sievenpiper HIS increases the front-to-back ratio because of the shielding effect, while simultaneously narrows the beamwidth due to the constructive interference. Moreover the unwanted cross polarized component is kept very low as in the case when using the microwave absorber. Only at wider angles a deviation between the two curves with a $5 \mathrm{~dB}$ better performance for the microwave absorber has to be mentioned concerning the suppression of the cross polarized component. But due to a cross polarization discrimination of better than $15 \mathrm{~dB}$ for the combination of antenna and HIS in the whole primary half space, this effect is of less importance. Furthermore, the pattern measured with the absorber reveals ripple for the cross polarized component in the upper horizontal diagramm which is founded in induced currents radiating from the fi- 
nite groundplane edges whereas the use of the HIS shows a smooth shape due to suppressing this currents and surface waves, respectively.

\section{Conclusions}

In this paper we presented the theory for describing a new kind of spatial filter known as Sievenpiper HIS. The unique in-phase reflection characteristics offered by these composite metallo-dielectric structures were explained and the well known design formulas based on the transmission-line theory were presented. The accuracy of the describing equations as well as the predicted filter properties have been verified by means of results derived from simulation models in the 3D fullwave solver $H F S S$ of Ansoft Corporation. Furthermore, the influence of the introduced HIS in conjuction with a reference antenna has also been stated by use of the simulation tool H F SS. Especially the input matching and the absolute antenna gain has been evaluated. Here also some parasitic effects occuring due to interaction in the reactive antenna near filed have been explained. Finally a prototype HIS was fabricated and measurements have been done where a planar broadband log.-per. four-arm trapezoidal antenna was placed over the prototype. The derived results in terms of arm input impedance over frequency as well as relative radiation patterns at a specific frequency were stated and evaluated. All the derived results showed good agreement with the expected behaviour based on the theoretical description of the HIS with only small deviations.

\section{References}

Clavijo, S.: Design Methodology for Sievenpiper High-Impedance Surfaces: An Artificial Magnetic Conductor for Positive Gain Electrically Small Antennas, IEEE Transactions on Antennas and Propagation, 51, 2678-2690, 2003.

Collin, R. E.: Field Theory Of Guided Waves, IEEE Press, New York, 1991.

King, R., Thiel, D., and Park, K.: The synthesis of surface reactances using an artificial dielectric, IEEE Transactions on Antennas Propagation, 31, 471-476, 1983.

Klemp, O., Schultz, M., and Eul, H.: Novel Logarithmically Periodic Antennas for Broadband Polarization Diversity Reception, AEÜ International Journal on Electronics and Communications, 1, 317-326, 2005.

Marcuvitz, N.: Waveguide Handbook, Peter Peregrinus Ltd, London, 1986.

Merill, W. M.: Effective Medium Theories for Artificial Materials Composed of Multiple Sizes of Spherical Inclusions in a Host Continuum, IEEE Transactions on Antennas and Propagation, 47, 142-148, 1999.

Rotman, W.: Plasma Simulation by Artificial DIelectrics and Parallel-Plate Media, IRE Transactions on Antennas and Propagation, 82-95, 1962.

Sievenpiper, D. F.: High-Impedance Electromagnetic Surfaces, Dissertation, University of California, Los Angeles, 1999.

Sievenpiper, D. F.: High-Impedance Electromagnetic Surfaces with a Forbidden Frequency Band, IEEE Transactions on Microwave Theory and Techniques, 51, 2059-2074, 2003.

Simovski, C. R.: High-Impedance Surfaces Having Stable Resonance With Respect to Polarisation and Incidence Angle, IEEE Transactions on Antennas and Propagation, 53, 908-914, 2005.

Veselago, V.: The electrodynamics of substances with simultaneously negative values $\epsilon$ and $\mu$, Sov. Phys. Uspheki, 10, 509-514, 1968.

Zinke, O. and Brunswig, H.: Hochfrequenztechnik 1, Springer Verlag, Berlin, 2000. 